\title{
Risk Level of Asthma and Chronic Obstructive Pulmonary Disease Through Design of an Intelligent Type-2 Fuzzy Expert System
}

\author{
Krishna Anand Sundararaman ${ }^{1 *}$, Vijayalakshmi Seerkalan ${ }^{2}$, Kalpana Rangarajan $^{3}$ \\ ${ }^{1}$ Department of CSE, SNIST, Hyderabad 501 301, India \\ ${ }^{2}$ M. Tech. (IT), College of Engineering, Guindy, Chennai 600 025, India \\ ${ }^{3}$ Graduate Engineer Trainee, Schneider Electric, Bangalore 560 100, India
}

Corresponding Author Email: krishnaanads@ @reenidhi.edu.in

https://doi.org/10.18280/i2m.180610

Received: 10 August 2019

Accepted: 16 October 2019

\section{Keywords:}

Type-2 fuzzy logic, expert systems, asthma, chronic obstructive pulmonary

disease, uncertainty, user interface

\begin{abstract}
Diagnosis of Asthma during the early stages plays a primary role in providing effective medical treatment. Different opinions may be given by different doctors for a patient having the same set of symptoms. This opinion is based on the type or degree of the disorder. There can be scenarios where the same doctor may diagnose differently for two different patients having similar symptoms. Hence, it can be concluded that the thought process of a doctor is fuzzy in nature. In any medical field, accuracy of diagnosis is extremely important as it concerns the life of a patient. Besides, with increase in number of hospitals and staff, the amount of measured data is extremely huge. Off late, mistakes have tended to creep during recording of measurements. Mistakes include carelessness, inaccurate measurements and failure in recording take place from time to time. Taking these points into consideration, the focus of the work has shifted to providing an improvement in the accuracy levels of detection. In this connection, a Type-2 Fuzzy model has been designed.
\end{abstract}

\section{INTRODUCTION}

There are numerous expert systems that have been designed to diagnose the severity of Asthma. The amount of data to be worked with is exponentially large [1]. Most of these systems focus on certain set of parameters which are considered to be the key [2-4]. However, a large number of parameters have been ignored. For instance, the minor day-to-day symptoms which in turn could play a decent role have been omitted from further study. Also, in certain cases, spirometry test results have been overlooked in the diagnosis.

To make the system more effective, a Fuzzy expert System had been designed that takes into consideration wheeze, cough, age, frequency of smoking, gender, rate of respiration, fever, night time awakenings, Body Mass Index (BMI), difficulty in speaking, usage of chemicals on a large scale near residence or place of work, pre-menstrual syndromes and chest tightness. Along with these factors, inference of severity of the disease is supported highly by the results obtained from the lung function tests conducted by the doctor.

Four intelligent controllers have been developed to perform detailed diagnosis. In turn, the complexity of the expert system has been reduced. Each controller takes into its system, a set of inputs (symptoms) and produces the severity of Asthma and Chronic Obstructive Pulmonary Disease (COPD) as output [57]. A membership function is drawn for each parameter that is considered as a part of the intelligent controller. Each controller has a set of rules to determine the severity of the symptoms and to produce an output corresponding to the patient's knowledge on his symptoms. The intermediate results obtained from the four intelligent controllers are further validated along with spirometry results and non-fuzzy parameters to obtain the severity of the disease. Medication and general guidelines are provided based on the results obtained from the expert system. The results of the same have been published earlier.

With accurate diagnosis being the chief focus in Medical areas, enough emphasis is provided to push the accuracy levels towards $100 \%$. Type-2 Fuzzy expert system goes a long way in achieving this purpose.

\section{LITERATURE SURVEY}

The term Fuzzy logic was proposed by Lofti A. Zadeh in the year 1965. Unlike the classical set, which takes either true or false as its value, fuzzy set can have a truth value ranging between 0 and 1. Fuzzy logic is more human intuitionist as it depends on the degree of truth and thereby makes use of linguistic variables.

Type-2 Fuzzy Logic was introduced by Zadeh in the year 1975, which was further enhanced by Mendel and Karnik. When the system consists of noisy data, Type-2 Fuzzy Logic Sets can yield a better performance than Type-1 Fuzzy logic.

A great deal of research is being conducted on real world applications using Fuzzy Logic. However, the trend in more recent times has shifted to the usage of Type 2 Fuzzy Logic owing to its excellent handling of uncertainties and missing information. Masaharu Mizumoto and Kokichi Tanaka, in the year 1976, studied about the various operations like join, meet and negation performed on Type-2 Fuzzy sets. In the year 1998, Karnik and Mendel designed a robust fuzzy expert system that can handle uncertainties and published their work. This work also focusses on type-reduction, set operations and properties of membership grades on Type- 2 sets, their relations and compositions along with defuzzification 
methodologies. They used their theory on Type-2 Fuzzy logic in practice in forecasting time series in the year 1999. They also demonstrated the improved performance by Type-2 Fuzzy system over Type-1 Fuzzy logic. The various uncertainty measures like cardinality, fuzziness, variance and skewness were discussed by Mendel and $\mathrm{Wu}$ in the year 2007. Various experiments were conducted on intelligent controllers to the study the nature of uncertainty in both Type- 1 and Type- 2 Fuzzy logic by Roberto Sepúlveda, Oscar Castillo, Patricia Melin, Antonio Rodríguez-Díaz, and Oscar Montiel in the latter part of the year. They concluded that the controllers produced enhanced performance on a Type-2 System. A team consisting of Fazel Zarandi, Rezaee, Turksen and Neshat designed a rule-based Type-2 Fuzzy expert system in the year 2009 to analyse the stock prices during the trading period.

The enhanced features provided by Type - 2 Fuzzy systems make its presence felt in real world applications. Its usage in medicine has provided an added fillip to the health industry.

\section{EXPERT SYSTEM}

An intelligent program has been designed that uses knowledge and inference procedures in detecting symptoms pertaining to asthma. This problem could not be solved by a conventional program due to the lack of an efficient algorithm. Expert systems have been successful in dealing with realworld problems that conventional programming methodologies have been unable to solve, especially those dealing with uncertain or incomplete information. Expert systems are generally designed differently from conventional programs because the problems usually have no algorithmic solutions and rely on inferences to achieve a reasonable solution. The process of developing an expert system has an indirect benefit since the knowledge of human experts must be put into an explicit form for entering into the computer. Since the knowledge is explicitly known, it could be examined for correctness, consistency and completeness. Besides, expert systems are also capable of amplifying human expertise.

\section{FUZZY EXPERT SYSTEM}

In order to overcome the difficulty of providing exact inputs, the concept of fuzzy expert system has been proposed. This system is able to handle uncertainty to a very fine extent. A Type-1 Fuzzy expert system had been designed which performs conflict resolution and produces good results. The work has been given its due recognition and in turn has been published in World Applied Sciences Journal [8]. To handle uncertainties much better, a Type-2 Fuzzy Expert System has been constructed. In many real life models, the data provided will be objective that is they would have a definite value. However, parameters which are not measured accurately will have only subjective values like high and large. Direct computations cannot be performed with such data. The advantage of the fuzzy model lies in the fact that subjective values can be provided apart from providing the membership functions in detail.

\section{HANDLING OF UNCERTAINTIES}

The Type 2 Fuzzy Expert System designed is able to handle the following uncertainties
1. Uncertainties associated with measurements and analysis

2. Uncertainties associated with the pattern, behaviour and context of the asthma patient

3. Inter and Intra User uncertainties where the opinion of different experts vary. Apart from that the mood of experts could also lead to various conflicts.

4. A few linguistic uncertainties may arise leading to ambiguities in the meanings.

Among the four, the occurrences of the first three are present in a substantial measure in the application taken into consideration. However, the system is able to make an accurate prediction despite the presence of these uncertainties $[9,10]$. Therefore, the design of the system acts as a balm in assisting the information from the doctors about diagnosis [11]

\section{COMPONENTS OF THE EXPERT SYSTEM}

\subsection{Knowledge base}

A knowledge base is considered to be a collection of structured and unstructured information that is necessary for effective functioning of an Expert System. The designed system consists of various rules that help in diagnosing the severity of Asthma and COPD. These rules contain all the necessary factors that would be considered by a medical expert. In order to synchronize the set of rules with that of the real world, the rules designed are fuzzy. For instance, the knowledge base required for a specific intelligent controller that takes 'Nocturnal Symptoms', 'Fever', 'Wheeze' and 'Respiratory Rate' as its input and produces 'Asthma', 'COPD' and 'Tuberculosis' as output has the knowledge base as shown in Figure 1.

\subsection{Reasoning engine}

The rules for each subsystem are given in such a way that, it handles the symptoms the patients are experiencing, in the manner by which a doctor would approach to diagnose the disease. Enough importance is given in assigning relevant weightages to rules that play a significant role and also assigning appropriate membership functions to them. This enhances the accuracy of the diagnosis obtained from the developed Expert System.

\subsection{Knowledge acquisition system}

The knowledge base required for the assessment of the disease was provided by a pulmonologist and an AsthmaCOPD camp conducted for about 50 patients. These patients experienced various symptoms that could affect the normal functioning of lungs. The results obtained from the medical expert, for the patients who reported with symptoms, are used in testing the developed Fuzzy Expert System. The characteristics of some of the patients varied to a large measure as compared to some other patients.

\subsection{Learning component}

The system needs to learn continuously. Learning is incorporated through modification in the knowledgebase. The choice of the rule to fire is based on conflict resolution strategy. The principle of resolution is carried out while deciding the most suitable rule. The set of relevant information is transmitted to working memory. The content of the rule base 
is matched with that of the working memory to decide the possible rules to be fired. This is shown in Figure 2. The consequences obtained in turn again modify the content of the working memory. In some cases, two or more rules are unified and a new consequent is obtained. This in turn has led to efficient forward chaining of rules.

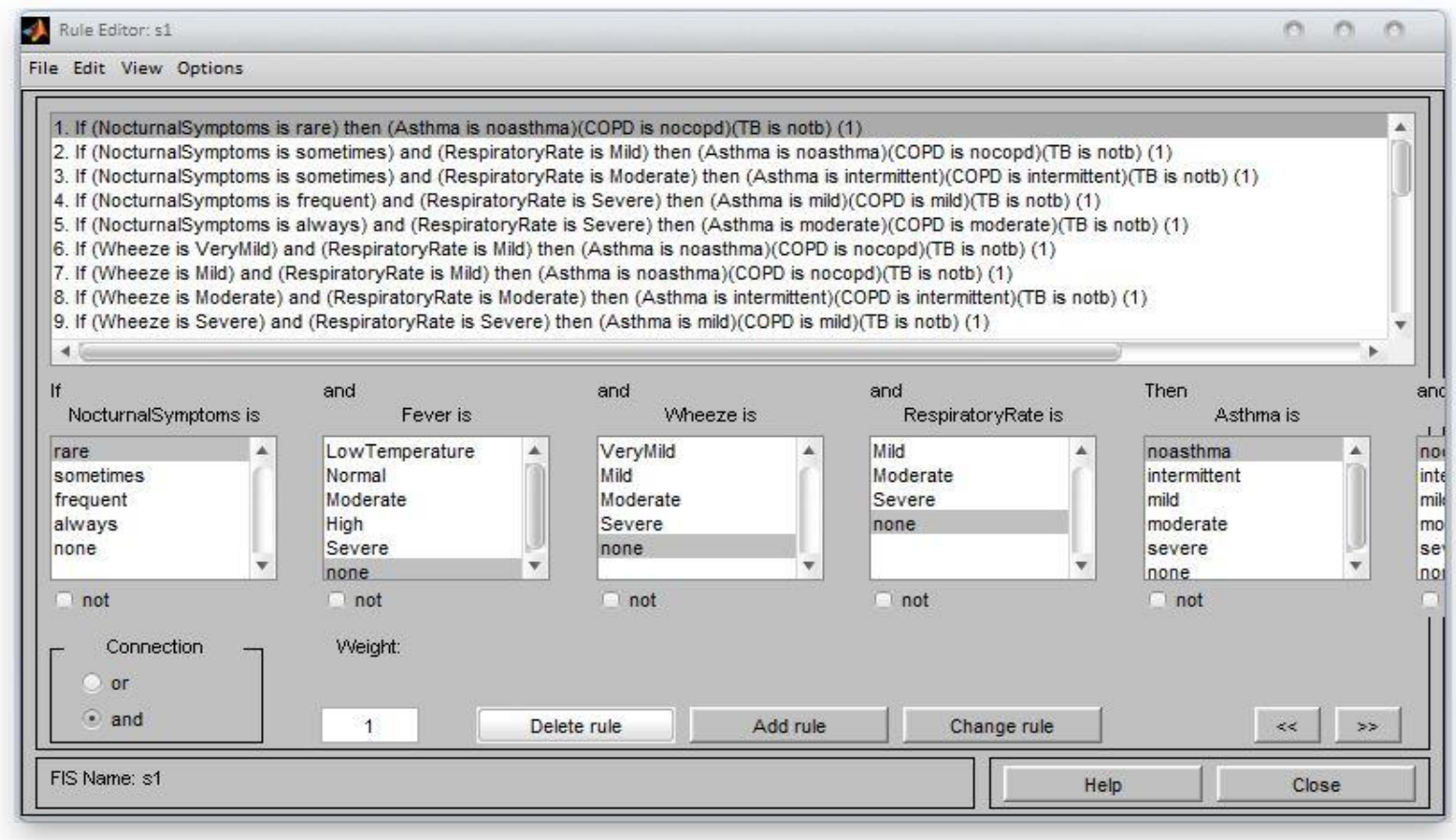

Figure 1. Knowledge base of an intelligent controller

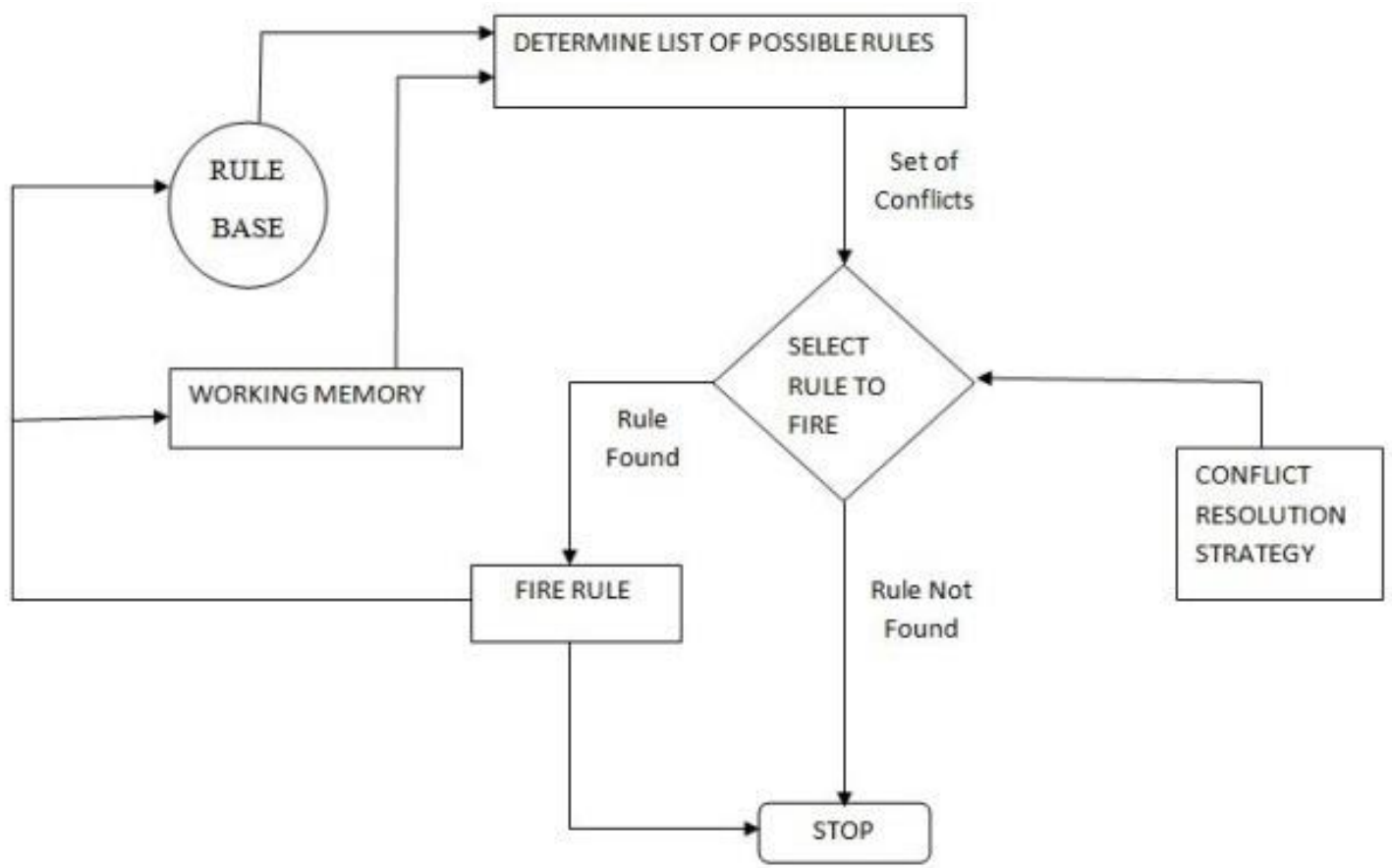

Figure 2. Rule chaining with conflict resolution

\subsection{Inference engine}

The fuzzy inference engine is able to find the firing level of each rule and finds the output of every rule. Besides, the outputs of all rules are aggregated together to determine the overall system output. The specialty of the system lies in its ability to handle conflicts and frame suitable conclusions. These conclusions in turn would help in framing a suitable decision.

\subsection{User interface}

The user interface implemented for the Fuzzy Expert System is a means of integrating both the fuzzy and non-fuzzy parameters considered in the detection of the levels of Asthma and COPD [12]. The User Interface of the Fuzzy Expert System is depicted in Figures $3 \mathrm{a}, 3 \mathrm{~b}$ and $3 \mathrm{c}$. Viewing details of existing patients have been provided in Figure 3a. Apart from that, new patients can also be added. Besides, a detailed 
report can also be generated. Figure $3 b$ deals with a Direct Manipulation interface where the details of a particular patient can be provided. The various symptoms suffered by the patient are supplied in Figure 3c.

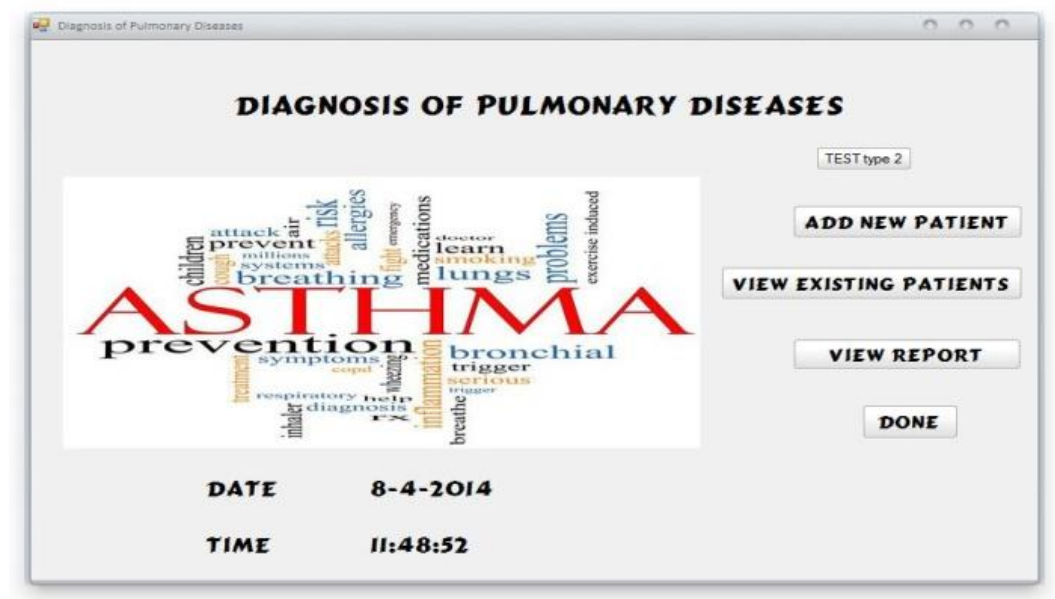

(a)

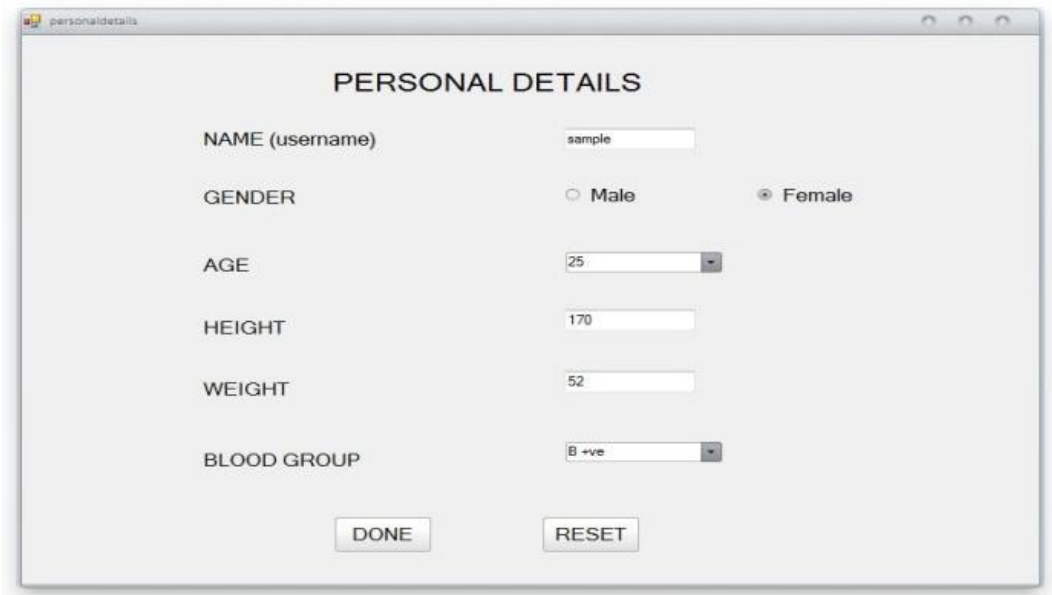

(b)

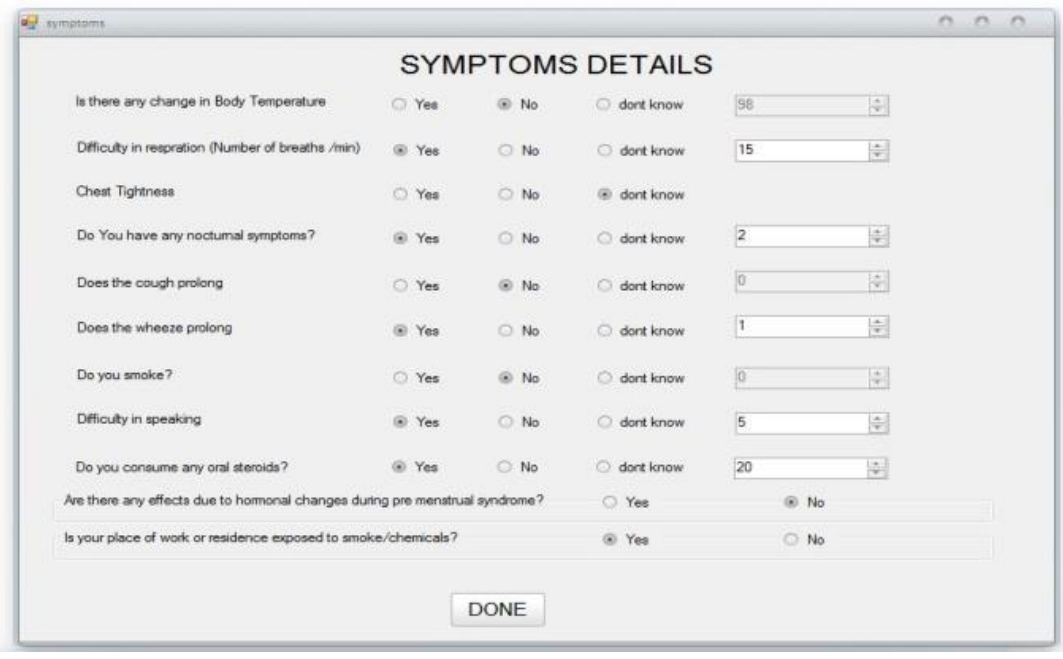

(c)

Figure 3. a) User interface for the fuzzy expert system; b) Interface to obtain patient's personal information; c) Interface to obtain patient's symptom details

\subsection{Defuzzification}

The centroid method is used here where a point representing the centre of gravity is found out. The advantage of the centroid method is that even a continuous function can be approximated to reach the level of arbitrary accuracy. Defuzzification deals with converting the fuzzy values to crisp sets.

\section{TYPE-2 FUZZY MODEL}

Type-2 Fuzzy Logic, as an extension to Type-1 Fuzzy sets, is helpful in situations where handling of uncertainties is a requisition. By handling uncertainties in a better manner, it provides an enhanced performance as opposed to Type-1 Fuzzy Logic in terms of accuracy. The footprint of uncertainty (FOU), in a Type-2 Fuzzy set, is bounded by two Type-1 
membership functions, an upper and a lower membership function as depicted in Figure 4.

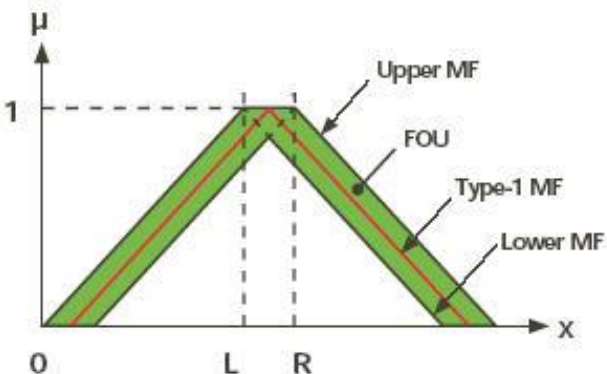

Figure 4. Type-2 membership function

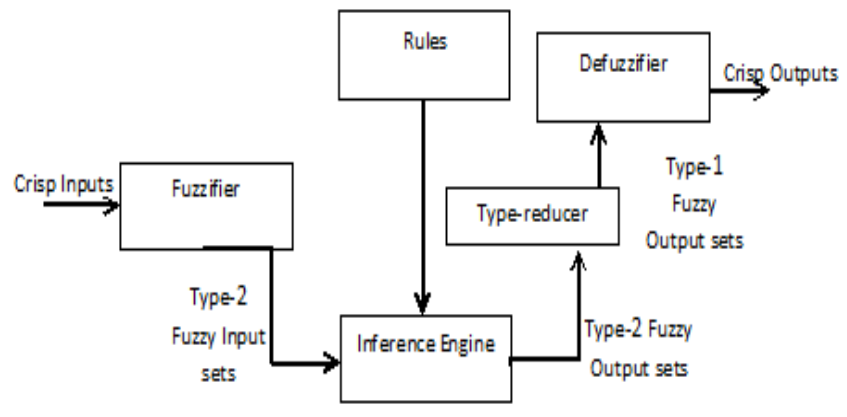

Figure 5. Type-2 fuzzy logic system block diagram

The Type-2 Fuzzy Logic System (FLS) includes a fuzzifier, a set of rules, an inference engine, a type-reducer and a defuzzifier as shown in Figure 5. The fuzzifier classifies the crisp inputs to its corresponding Type-2 Fuzzy set. The set of rules defined in the FLS have Type- 2 parameters as its antecedent and consequence. Based on the set of rules, the inference engine produces a fuzzy output for a given Type- 2 input. Type-reducer converts the obtained Type-2 Fuzzy output to a Type-1 Fuzzy parameter. For this to take place, the central area of the fuzzy set has to be taken into consideration. This is provided by centroid method. This method has been put to full use as and when the situation demanded during construction of fuzzy sets.

\section{TYPE-2 FUZZY PARAMETERS}

Type-2 Fuzzy Expert System provides a higher level of abstraction and is capable of handling more uncertainty. It considers only a limited number of fuzzy modifiers at a higher level and classifies them further upon necessity.

The fuzzy parameters considered for the design of Type-2 Fuzzy Controller are 'Age', 'Body Mass Index', 'Oral Steroids', 'Cough' and 'Wheeze'. These fuzzy parameters are considered due to their ambiguous nature. It has a varied perception among the patients. For instance, a patient who is health conscious may consider 'Normal' body mass index to range between 18 and 21 whereas, another patient may take the range 18 to 23 as 'Normal'. Hence, the term 'Normal' has various perceptions among the patients. In order to generalise the value among all patients, an upper and lower range of values is given for the parameters.

A Type-2 Fuzzy logic Toolbox is designed for the generation of Type-2 Fuzzy parameters as shown in Figure 6. The Toolbox developed is used to create membership functions for any Type- 2 variable. For any Type- 2 parameter, maximum of six membership functions could be drawn using the developed toolbox. The type of membership function can be triangular, trapezoidal, or a combination of both.

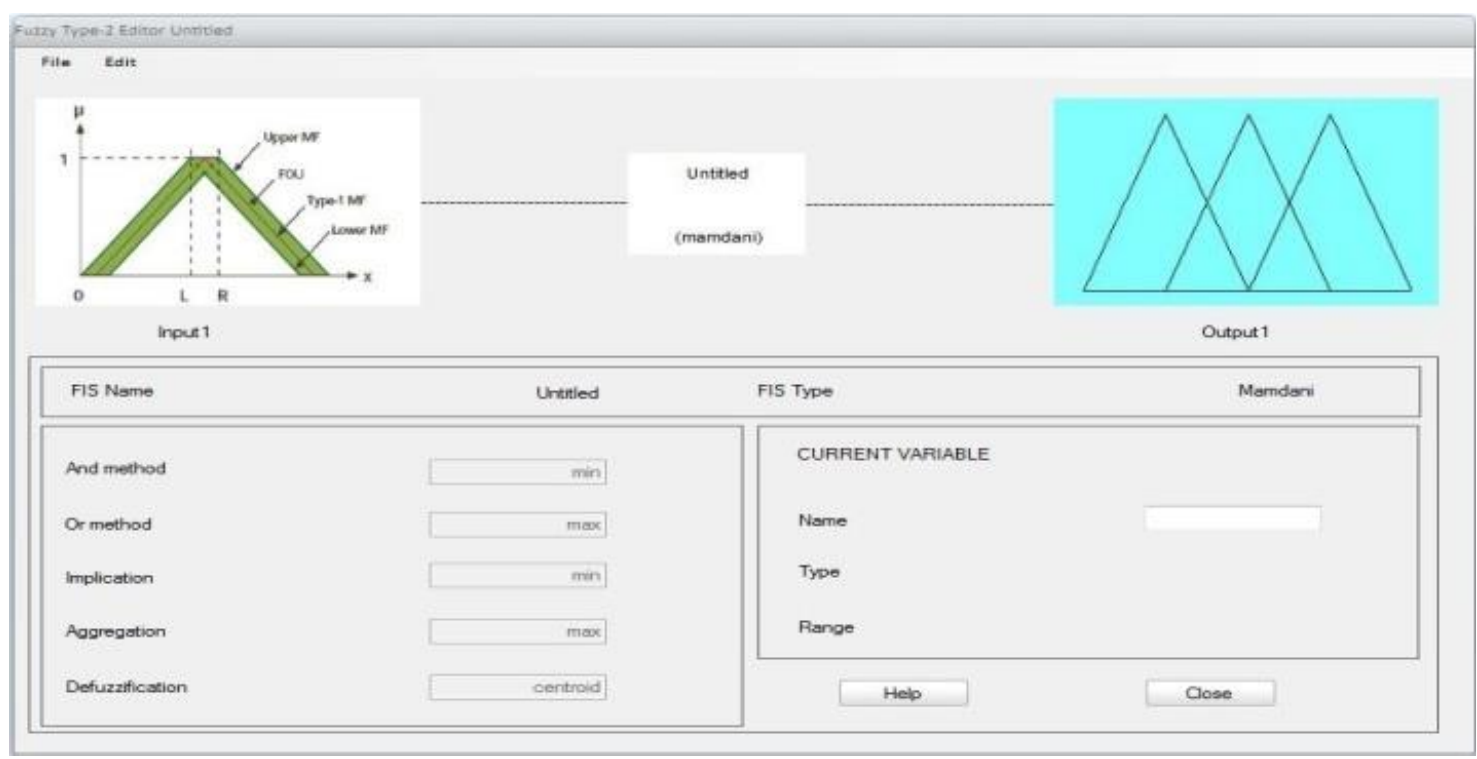

Figure 6. Type-2 fuzzy logic toolbox

\section{INCORPORATION OF TYPE-2 MODEL IN DIAGNOSIS}

Using the Type-2 Fuzzy logic toolbox, five Type-2 parameters namely 'Age', 'Body Mass Index', 'Oral steroids', 'Cough' and 'Wheeze' are created. The upper and lower membership boundaries for the parameter are given as input as shown in Figure 7. Based on the given input range of values for any particular parameter, the corresponding Type-2 membership graph is generated as shown in Figure 8.

Membership functions for the Type-2 parameter 'Age' is depicted in Figure 5. It consists of four parameters namely 
'child', 'youngster', 'middleage' and 'old'. In Type-1 Fuzzy model, the parameter 'child' can deal with any person whose age ranges from 0 to 19 . However, the level of truth varies. But for the range between 5 and 11, the truth value is ' 1 '. This range could vary among people. Hence, an upper and a lower range of limit are given to the parameter 'child'. The revised ranges for the upper and lower limit are 0 to 19 and 1 to 13 respectively. The value for 'child' between the ages 5 and 11 however continues to be perfectly true always. Consequently, a twelve year old in Type-1 Fuzzy model will have a degree of truthness of 0.6 and in a Type-2 Fuzzy model, the degree of truthness varies from 0.5 to 0.6 . This varied range of truth value is helpful in handling more uncertainty and in producing more precise results.

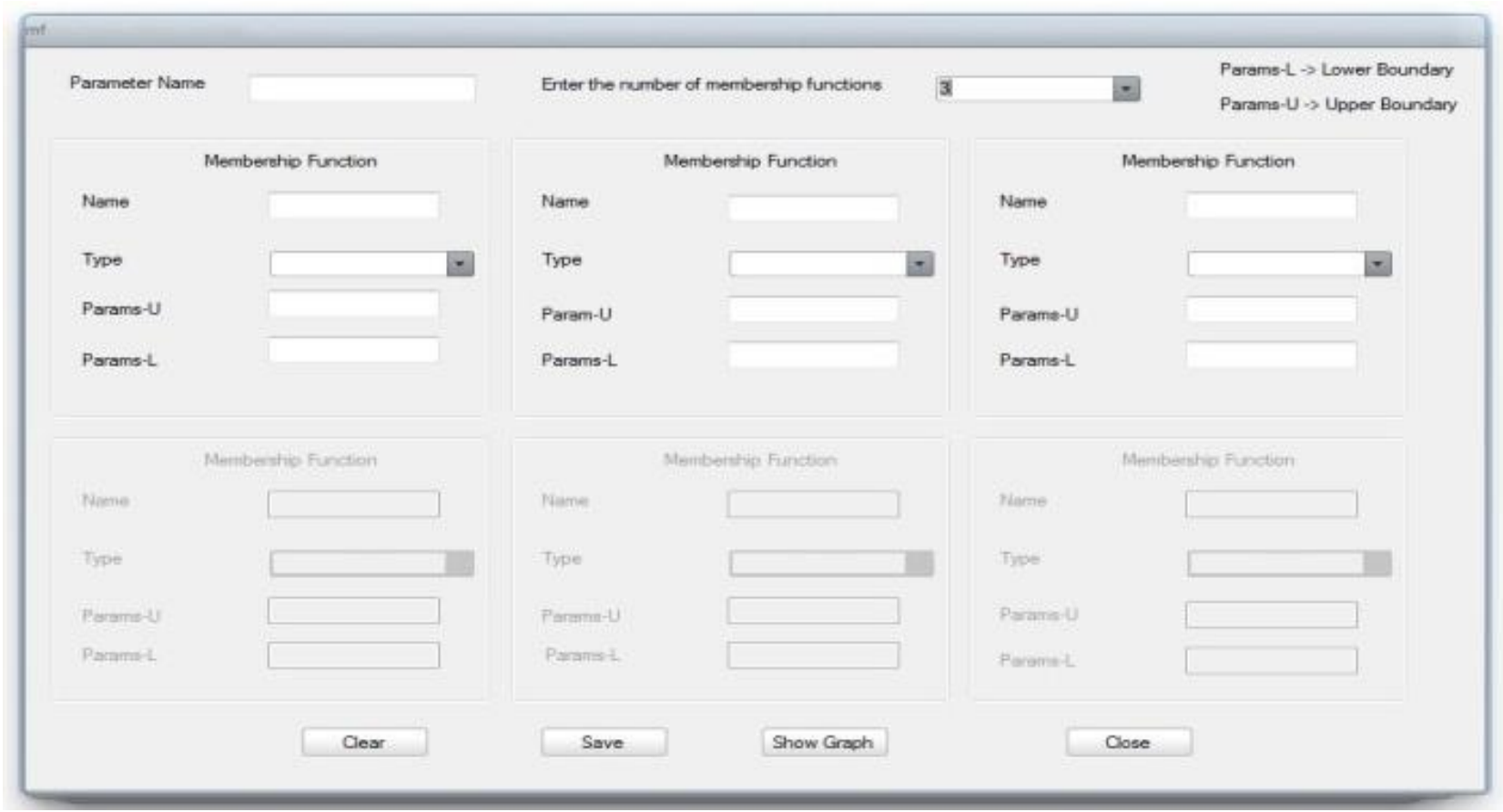

Figure 7. Membership function input

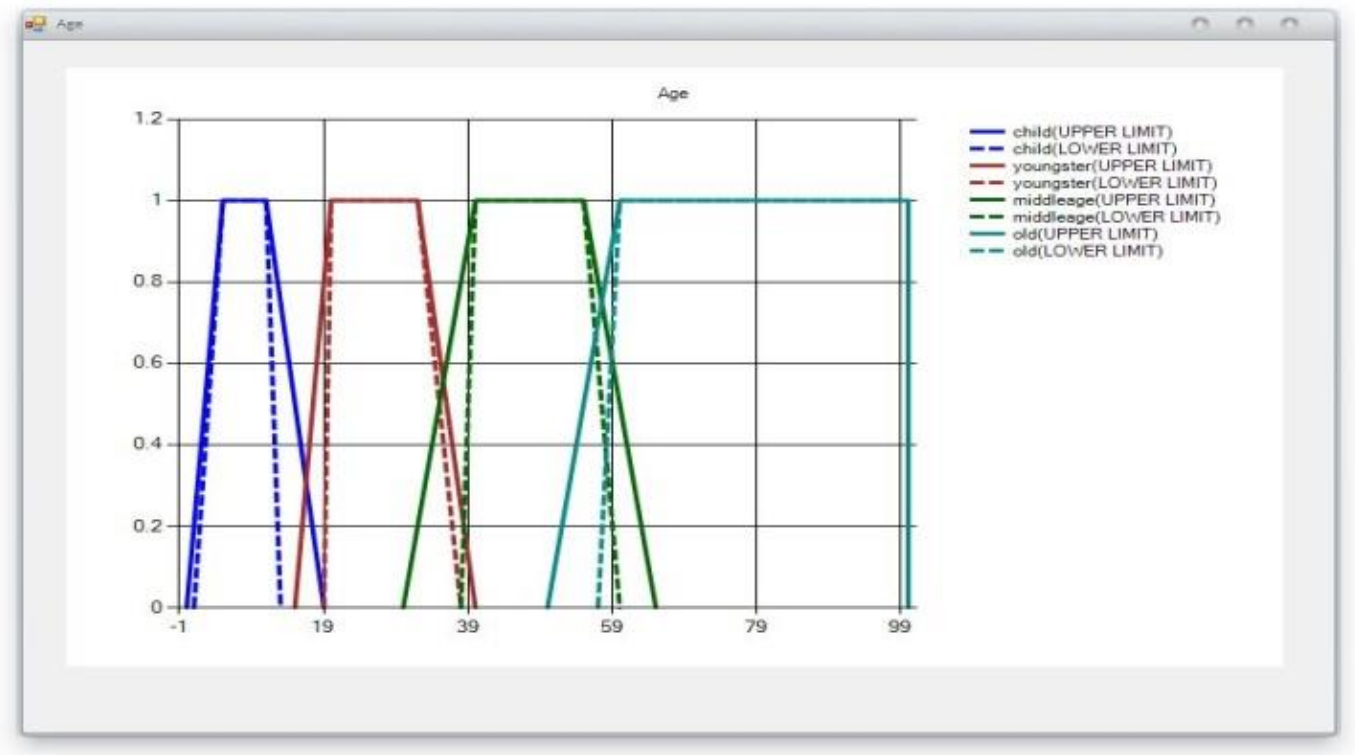

Figure 8. Type-2 Fuzzy parameter - 'Age'

\section{CONVERSION FROM TYPE-2 TO TYPE-1}

The Type- 2 parameter has to be converted to an appropriate Type-1 parameter for further analysis. The conversion to a Type-1 parameter is based on the centroid method. As discussed in Section 8, a twelve year old child has a degree of truth ranging from 0.5 to 0.6 . Hence the centroid value 0.55 is used for further process.
Initially, the user input value for any Type-2 parameter is classified to its corresponding membership variable. For the user specified input, there are two truth values and hence we reach at a consolidated value based on centroid method. The membership function for the required variable is redrawn in Type-1 Fuzzy model, to improve the accuracy. The altered membership functions in various intelligent controllers are used to identify the severity of the disease. 
The membership functions for the parameters are not improved in all cases. It is altered only in scenarios where the normalised value obtained from centroid method does not match with the existing membership value for the same input in Type-1 Fuzzy logic.

\section{COMPARATIVE ANALYSIS}

To analyse the advantage of Type-2 Fuzzy Logic over Type1 Expert System, a sample patient is considered to view the difference in results. A 25 year old patient (female, height$170 \mathrm{~cm}$ and weight-52kg) with normal body temperature and respiratory rate is taken into consideration. The patient does not suffer from chest tightness, difficulty in speaking and nocturnal symptoms. The patient has cough that prolongs for two days and wheeze for three days. The patient takes in a very less dosage of oral steroids and resides in a place where chemicals are exposed in a huge amount. The spirometry details of the patient include ' $73 \% \mathrm{FVC}$ ', ' $78 \% \mathrm{FEV} 1$ ' and ' $92 \%$ FEV/FVC'. The patient is likely to have 'Moderate Asthma' which ranges from 55 to 85 considering the severity of the disease on a scale of 1 to $100[13,14]$. The difference in outputs obtained from Type-1 and Type-2 Fuzzy Logic System is highlighted as shown in Figure 9. A sample patient report is shown in Figure 10.

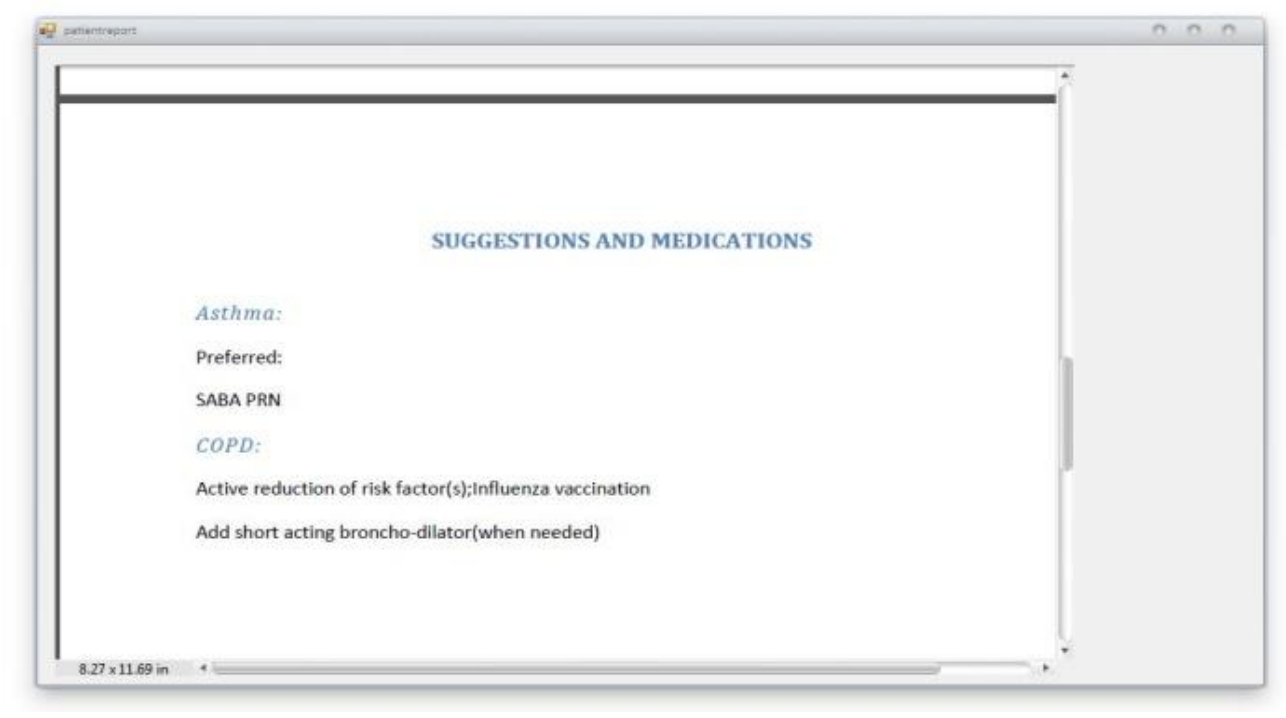

Figure 9. Comparison between Type-1 and Type-2 fuzzy logic output

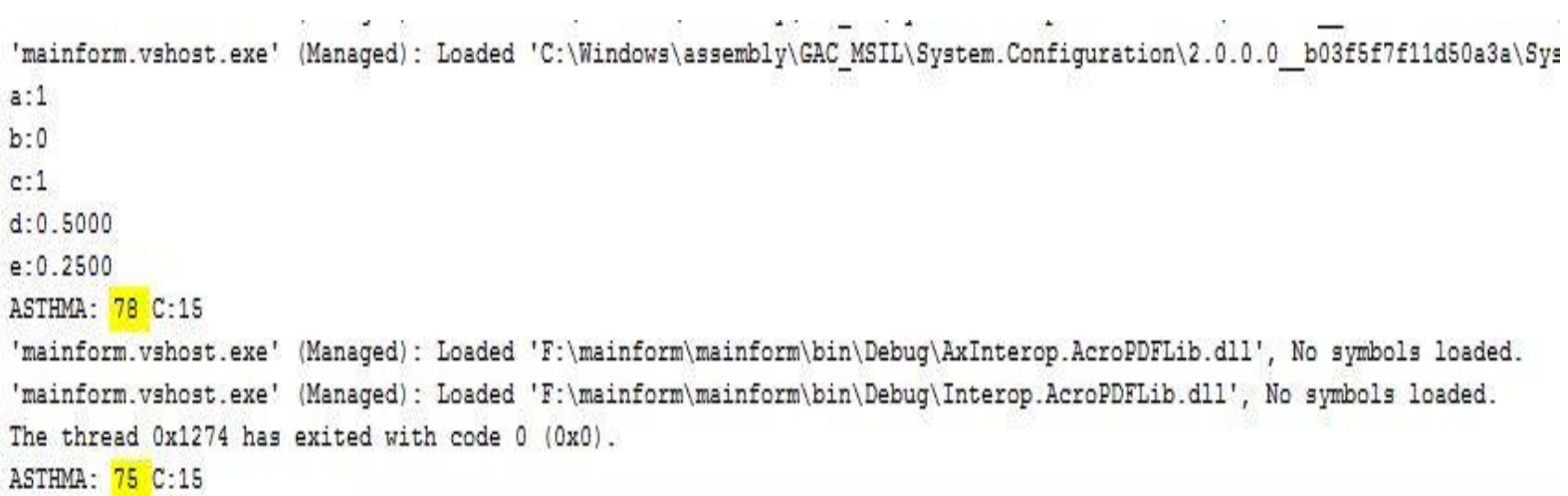

Figure 10. Sample patient report

\section{CONCLUSIONS AND RECOMMENDATIONS}

The Fuzzy Expert System estimates the levels of Asthma and COPD and produce suggestions based on the severity predicted by the system. The system considers all parameters essential for accurate estimation of the disease. The intensity of the treatment provided by the system depends on the severity of all parameters considered. The prescribed treatment is undergone by the patient for about two to six weeks and the patient is tested again for the severity of the disease to notice any improvements.

It could be easily observed that the designed system is able to make an excellent prediction of the amount of risk associated with asthma. The notable feature to be observed is that the values could be predicted much faster and an expert may not be needed at that point of time. The fact that the results match expert diagnosis opens up a new frontier in the usage of Soft Computing in medical applications [15]. Human errors and omissions which could have led to catastrophical events are no longer a cause of worry.

The fact that the designed system's efficiency is high is no longer under scrutiny. Even though the designed system has acted as a panacea in places where there could be scarcity of medical experts, other learning techniques could also be tried out. The feasibility of system incorporating learning using identification trees, genetic algorithms, k-decision trees and 
analogies could also be explored. The results obtained from those techniques could provide a new dimension in the field of medical research

\section{REFERENCES}

[1] Zolnoori, M., Zarandi, M.H.F., Moin, M., Taherian, M. (2010). Fuzzy rule-based expert system for evaluating level of asthma control. Journal of Medical Systems, 36(5): 2947-2958. https://doi.org/10.1007/s10916-0119773-3

[2] Lurie, A., Marsala, C., Hartley, S., Bouchon-Meunier, B., Dusser, D. (2007). Patient's perception of asthma severity. Respiratory Medicine, 101(10): 2145-2152. https://doi.org/10.1016/j.rmed.2007.05.027

[3] Lomotan, E.A., Hoeksema, L.J., Edmonds, D.E., Ramirez-Garnica, G., Shiffman, R.N., Horwitz, L.I. (2011). Evaluating the use of a computerized clinical decision support system for asthma by paediatric pulmonologists. International Journal of Medical Informatics, $\quad 81(3)$ : 157-165. https://doi.org/10.1016/j.ijmedinf.2011.11.004

[4] Zolnoori, M., Zarandi, M.H.F., Moin, M. (2010). Fuzzy rule-based expert system for evaluation possibility of fatal asthma. Journal of Health Informatics in Developing Countries, 36: 2947-2958. https://doi.org/10.1007/s10916-011-9773-3

[5] Er, O., Yumusak, N., Temurtas, F. (2010). Chest diseases diagnosis using artificial neural networks. Expert Systems with Applications, 37(12): 7648-7655. https://doi.org/10.1016/j.eswa.2010.04.078

[6] Er, O., Yumusak, N., Temurtas, F. (2012). Diagnosis of chest diseases using artificial immune system., Expert Systems with Applications, 39(2): 1862-1868. https://doi.org/10.1016/j.eswa.2011.08.064

[7] Krishna Anand, S., Suganya, C., Padmapriya, N., Vignesh, G. (2011). Designing a fuzzy expert system for personal diabetic diet recommendation. National Conference on Recent Advances in Computer Vision and Information Technology, NCVIT'11, pp. 545-550.

[8] Krishna Anand, S., Kalpana, R., Vijayalakshmi, S. (2013). Design and Implementation of a fuzzy expert system for detecting and estimating the level of asthma and chronic obstructive pulmonary disease. World Applied Sciences Journal, 23(2): 213-223. https://doi.org/10.5829/idosi.wasj.2013.23.02.13046

[9] Phillips-Wren, G., Sharkey, P., Dy, S.M. (2008). Mining lung cancer patient data to assess health care resource utilization. Expert Systems with Applications, 35(4): 1611-1619. https://doi.org/10.1016/j.eswa.2007.08.076

[10] Dexheimer, J.W., Abramo, T.J., Arnold, D.H., Johnson, K.B., Shyr, Y., Ye, F., Fan, K.H., Patel, N., Aronsky, D. (2013). An asthma management system in a paediatric emergency department. International Journal of Medical Informatics, $\quad 82(4)$ : 230-238. https://doi.org/10.1016/j.ijmedinf.2012.11.006

[11] Chakraborty, C., Mitra, T., Mukherjee, A., Ray, A.K. (2009). CAIDSA: Computer-aided intelligent diagnostic system for bronchial asthma. Expert Systems with Applications, $\quad 36(3)$ : 4958-4966. https://doi.org/10.1016/j.eswa.2008.06.025

[12] Wu, Y.G., Wu, Y.M., Wang, J., Yan, Z., Qu, L.B., Xiang, B.R., Zhang, Y.G. (2011). An optimal tumor marker group-coupled artificial neural network for diagnosis of lung cancer. Expert Systems with Applications, 38(9): 11329-11334. https://doi.org/10.1016/j.eswa.2011.02.183

[13] Flores-Fernandez, J.M., Herrera-Lopez, E.J., SanchezLlamas, F., Rojas-Calvillo, A., Cabrera-Galeana, P.A., Leal-Pacheco, G., Gonzalez-Palomar, M.G., Femat, R., Martinez-Velazquez, M. (2012). Development of an optimized multi-biomarker panel for the detection of lung cancer based on principal component analysis and artificial neural network modelling. Expert Systems with Applications, $\quad 39(12)$ : $\quad$ 10851-10856. https://doi.org/10.1016/j.eswa.2012.03.008

[14] Ucar, T., Karahoca, A., Karahoca, D. (2012). Tuberculosis disease diagnosis by using adaptive neuro fuzzy inference system and rough sets. Neural Computing and Applications, 23: 471-483. https://doi.org/10.1007/s00521-012-0942-1

[15] Anand, K., Mamatha, E., Reddy, C.S., Prabha, M. (2019). Design of neural network based expert system for automated lime kiln system. Journal Européen des Systèmes Automatisés, 52(4): 369-376. https://doi.org/10.18280/jesa.520406 